\title{
Lattice Calculation of the Hadronic Light by Light Contributions to the Muon Anomalous Magnetic Moment
}

\section{Luchang Jin*}

Physics Department, Columbia University, New York, NY 10027, USA

E-mail: 1 j2289@columbia.edu

\begin{abstract}
The anomalous magnetic moment of muon, $g-2$, is a very precisely measured quantity. However, the current measurement disgrees with standard model by about 3 standard deviations. Hadronic vaccum polarization and hadronic light by light are the two types of processes that contribute most to the theorectial uncertainty. We will present the light by light lattice calculation and some strategies of including QED on lattice.
\end{abstract}

The 32nd International Symposium on Lattice Field Theory,

23-28 June, 2014

Columbia University New York, $N Y$

* Speaker. 


\section{Introduction}

The anomalous magnetic moment of muon defined in terms of the photon-muon vertex function.

$$
\bar{u}\left(p^{\prime}\right) \Gamma^{\mu}\left(p^{\prime}, p\right) u(p)=\bar{u}\left(p^{\prime}\right)\left[F_{1}\left(q^{2}\right) \gamma_{\mu}+i \frac{F_{2}\left(q^{2}\right)}{4 m}\left[\gamma_{\mu}, \gamma_{v}\right] q_{v}\right] u(p)
$$

Here $F_{2}(0)=(g-2) / 2$. The value has been measured very precisely by BNL E821 [1]. It can also be calculated theoretically to great precision as well [3]. The 3 standard deviations between the experiment and theory makes this value very interesty. The value from a much more accurate experiment Fermilab E989 is expected in few years.

We will focus on the lattice calculation of connected hadronic light by light amplitude, which is the second largest theoretical uncertainty contributor and the only current available estimate is by model [6]. This subject was begun by T. Blum, M. Hayakawa, T. Izubuchi more than 5 years ago [5][2]. Below are the Feynman diagrams for this process.

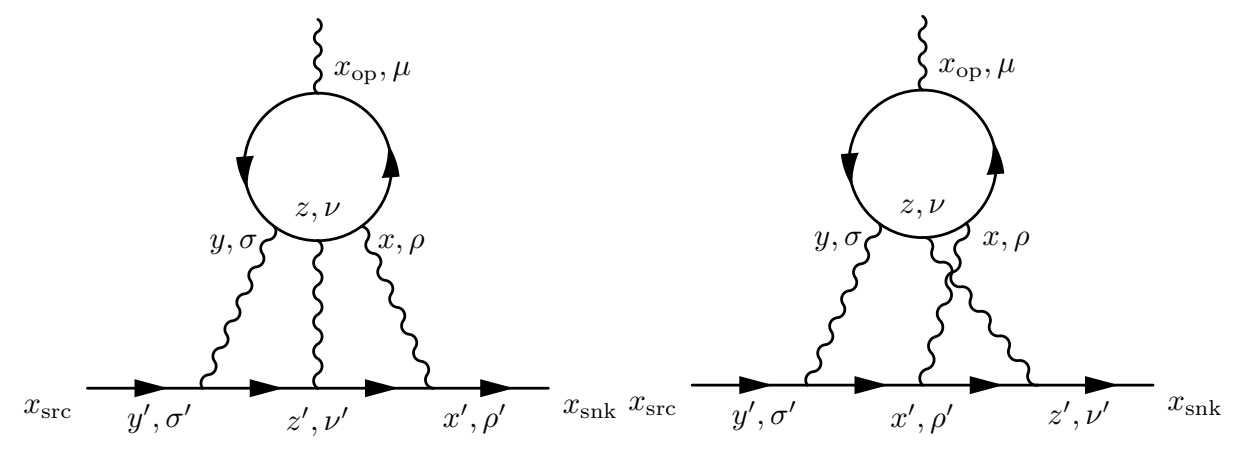

Figure 1: Light by Light diagrams. There are 4 other possible permutations.

To calculate this process, we need to include QED in lattice simulations. We will start with a simpler diagram which is the major contributor of the muon anomalous magnetic moment.

\section{Lattice QED with Schwinger Term as an Example}

We would like to do a standard Euclidean-space lattice calculation with a muon source and sink, well separated in Euclidean time, computing the following amplitude

$$
\mathscr{M}_{\mu}^{1-\text { loop }}=(-i e)^{2} \sum_{x, x^{\prime}} S\left(x_{\mathrm{snk}}, x\right) \gamma_{v} S\left(x, x_{\mathrm{op}}\right) \gamma_{\mu} S\left(x_{\mathrm{op}}, x^{\prime}\right) \gamma_{v^{\prime}} S\left(x^{\prime}, x_{\mathrm{src}}\right) G_{v v^{\prime}}\left(x, x^{\prime}\right)
$$

Where $S(x, y)$ is the muon propagator and $G_{\mu v}(x, y)$ is the photon propagator. For brevity, we use a local current everywhere in the above formula, but in our actual implementation the muon interacts with the QED gauge field via the gauge link $U_{\mu}(x)=\exp \left(i e A_{\mu}(x)\right)$, so the internal photon is coupled to the conserved vector current.

Naively, the sum would require $\mathscr{O}\left(\right.$ Volume $\left.^{2}\right)$ computation, which is not affordable. We discuss two strategies: 
- Exact Photon: Fast Fourier Transformation.

- Stochastic Photon: Calculate the sum stochastically.

Both approaches make the problem $\mathscr{O}$ (Volume).

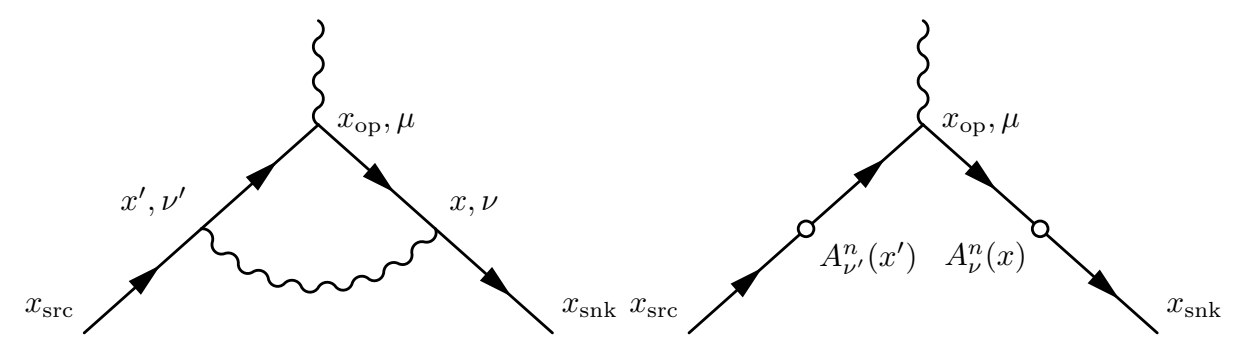

Figure 2: Schwinger term diagram with (L) exact photon (R) stochastic photon.

\subsection{Stochastic Photon}

Just as is done for lattice QCD, we can average over gauge configurations which include the electromagnetic field contribution. For example, the photon propagator can be evaluated approximately with $N$ stochastic samples:

$$
G_{\mu v}(x, y) \approx \frac{1}{N} \sum_{n=1}^{N} A_{\mu}^{n}(x) A_{v}^{n}(y) .
$$

We use Feynman gauge and a non-compact QED gauge action. This action is relatively simple so we can generate the properly distributed gauge field directly without using a Markov process. For brevity, we ignore the complexities caused by finite lattice spacing in the following formula:

$$
A_{\mu}^{n}(x)=\frac{1}{\sqrt{V}} \sqrt{2} \operatorname{Re} \sum_{k} \frac{\varepsilon_{\mu}^{n}(k)}{\sqrt{\left|k^{2}\right|}} e^{i k \cdot x}
$$

where the $\varepsilon_{\mu}^{n}(k)$ are random variables, which obey

$$
\frac{1}{N} \sum_{n=0}^{N} \varepsilon_{\mu}^{n}(k) \varepsilon_{v}^{n *}\left(k^{\prime}\right) \approx \delta_{\mu \nu} \delta_{k k^{\prime}}
$$

If we substitute the factorized approximate photon propagator expression into the original Schwinger term amplitude, we find

$$
\begin{aligned}
\mathscr{M}_{\mu}^{1 \text {-loop }} \approx & (-i e)^{2} \frac{1}{N} \sum_{n=1}^{N} \\
\cdot & {\left[\sum_{x} S\left(x_{\mathrm{snk}}, x\right) \gamma_{v} A_{v}^{n}(x) S\left(x, x_{\mathrm{op}}\right)\right] \gamma_{\mu}\left[\sum_{x^{\prime}} S\left(x_{\mathrm{op}}, x\right) \gamma_{v^{\prime}} A_{v^{\prime}}^{n}\left(x^{\prime}\right) S\left(x^{\prime}, x_{\mathrm{src}}\right)\right] . }
\end{aligned}
$$

The sum over $x, x^{\prime}$ can be then performed separately thus reducing the complexity to $\mathscr{O}$ (Volume). This approach is very general, and can be applied anywhere. However, this method can be very costly. It generally takes several thousands of samples to reduce the stochastic error to the percent level. 


\subsection{Exact Photon}

The photon propagator in momentum space is also a factorized formula.

$$
G_{\mu v}(x, y)=\frac{1}{V} \sum_{k} \frac{\delta_{\mu v}}{k^{2}} e^{i k \cdot(x-y)}
$$

We then rearrange the original formula so that the sum over $x, x^{\prime}$ can be performed separately.

$$
\begin{aligned}
\mathscr{M}_{\mu}^{1 \text {-loop }}= & (-i e)^{2} \frac{1}{V} \sum_{k} \frac{\delta_{v v^{\prime}}}{k^{2}} \\
\cdot & {\left[\sum_{x} S\left(x_{\mathrm{snk}}, x\right) \gamma_{v} e^{i k \cdot x} S\left(x, x_{\mathrm{op}}\right)\right] \gamma_{\mu}\left[\sum_{x^{\prime}} S\left(x_{\mathrm{op}}, x^{\prime}\right) \gamma_{v^{\prime}} e^{-i k \cdot x^{\prime}} S\left(x^{\prime}, x_{\mathrm{src}}\right)\right] }
\end{aligned}
$$

The expressions in brackets can be calculated with fast Fourier transformation. This method is very efficient and is completely free of stochastic noise. However, sometimes this method cannot be applied and we need to switch back to the stochastic photon method.

For the Schwinger term problem, this method fits perfectly. We use this method to study the finite volume and discretization errors, which could give us some insight into the light by light calculation.

\subsection{Finite Volume Effects and Discretization Errors}
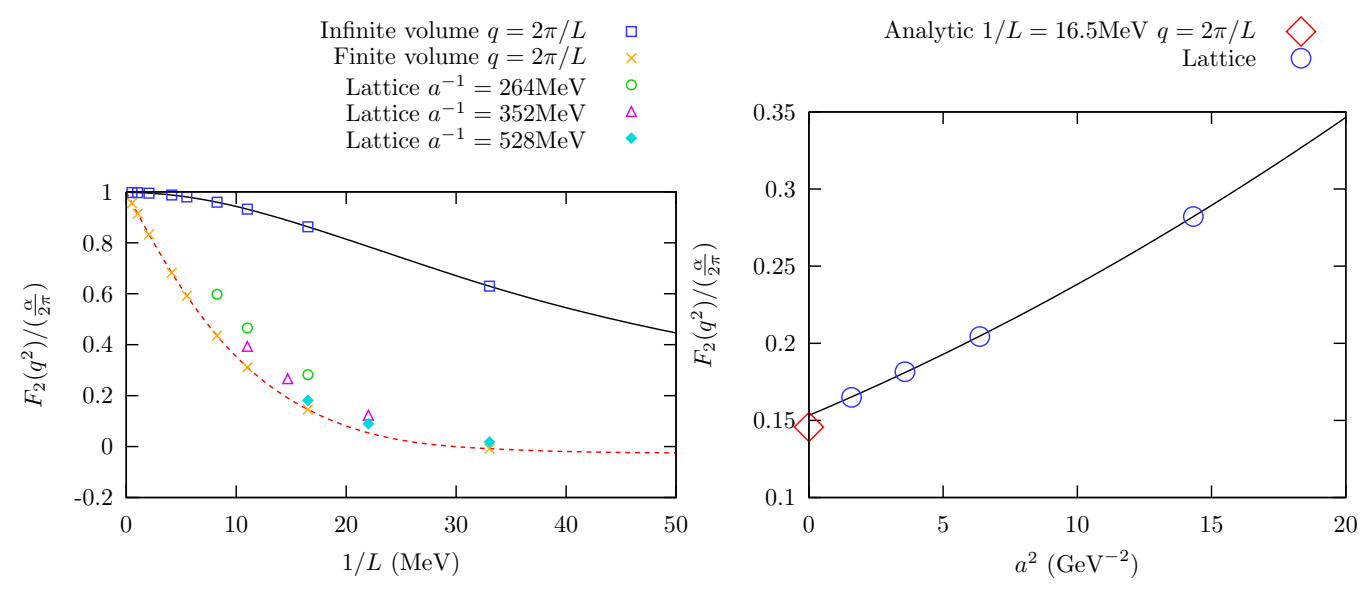

Figure 3: Lattice sizes are $16^{3} \times 64,24^{3} \times 96,32^{3} \times 128,48^{3} \times 192$ with $L_{s}=8$ and $t_{\mathrm{snk}}-t_{\mathrm{op}}=t_{\mathrm{op}}-t_{\mathrm{src}}=$ $T / 4$. The dashed line represents the analytic result in finite volume with momentum transfer $q=2 \pi / L$. The muon mass $m_{\mu}=105 \mathrm{MeV}$ is used to set the lattice spacing $a$. (L) The solid line represents the analytic result in infinite volume but the same non-zero momentum transfer. (R) The line shows the 2 nd order polynomial obtained by fitting the lattice results. An $a^{4}$ term is visible.

In order to compare with lattice simulation results, we also calculated the Schwinger term in finite volume and non-zero momentum transfer in the continuum limit. We calculated this "analytic" result using the analytic form of the muon propagator and doing the momentum sum numerically without a lattice cutoff. The leading term of the $1 / L$ expansion of our results agree with the NRQED calculation in [4]. 


\section{Connected Light by Light Evaluation Strategy}

There are three photons connected to the quark loop, the exact photon method cannot be applied to all of them. However, we can still treat one photon to be exact, as shown in Figure 4. Again, we use the local current everywhere in the formula below, but in our actual implementation the internal photons are coupled to the conserved vector current. ${ }^{1}$
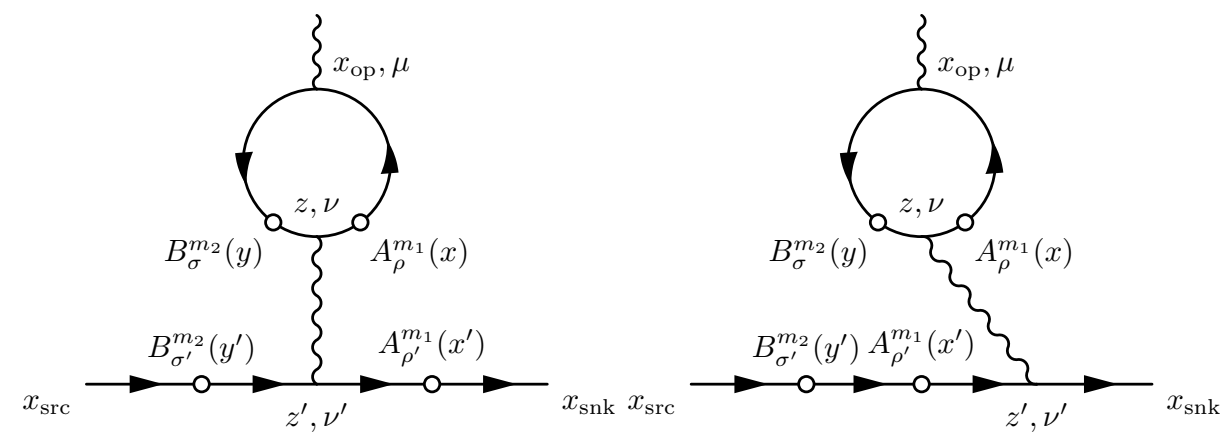

Figure 4: Light by Light diagrams calculated with one exact photon and two stochastic photon. There are 4 other possible permutations.

$$
\begin{aligned}
\mathscr{M}_{\mu}^{\mathrm{LbL}}= & -(-i e)^{6} \sum_{x, y, z} \operatorname{tr}\left[\gamma_{\mu} S_{q}\left(x_{\mathrm{op}}, x\right) \gamma_{\rho} S_{q}(x, z) \gamma_{v} S_{q}(z, y) \gamma_{\sigma} S_{q}\left(y, x_{\mathrm{op}}\right)\right] \\
\cdot & \sum_{x^{\prime}, y^{\prime}, z^{\prime}}\left[\frac{1}{M} \sum_{m_{1}=1}^{M} A_{\rho}^{m_{1}}(x) A_{\rho^{\prime}}^{m_{1}}\left(x^{\prime}\right)\right]\left[\frac{1}{M} \sum_{m_{2}=1}^{M} B_{\sigma}^{m_{2}}(y) B_{\sigma^{\prime}}^{m_{2}}\left(y^{\prime}\right)\right]\left[\frac{1}{V} \sum_{k} \frac{\delta_{v v^{\prime}}}{k^{2}} e^{\left.i k \cdot\left(z-z^{\prime}\right)\right]}\right. \\
\cdot & {\left[S\left(x_{\mathrm{snk}}, x^{\prime}\right) \gamma_{\rho^{\prime}} S\left(x^{\prime}, z^{\prime}\right) \gamma_{v^{\prime}} S\left(z^{\prime}, y^{\prime}\right) \gamma_{\sigma^{\prime}} S\left(y^{\prime}, x_{\mathrm{src}}\right)\right.} \\
& +S\left(x_{\mathrm{snk}}, z^{\prime}\right) \gamma_{v^{\prime}} S\left(z^{\prime}, x^{\prime}\right) \gamma_{\rho^{\prime}} S\left(x^{\prime}, y^{\prime}\right) \gamma_{\sigma^{\prime}} S\left(y^{\prime}, x_{\mathrm{src}}\right) \\
& + \text { other } 4 \text { permutations] } \\
= & -(-i e)^{6} \frac{1}{M^{2}} \sum_{m_{1}, m_{2}=1}^{M} \frac{1}{V} \sum_{k} \frac{\delta_{v v^{\prime}}}{k^{2}} \\
& \cdot \sum_{z} \operatorname{tr}\left\{\gamma_{\mu}\left[\sum_{x} S_{q}\left(x_{\mathrm{op}}, x\right) \gamma_{\rho} A_{\rho}^{m_{1}}(x) S_{q}(x, z)\right] \gamma_{v} e^{i k \cdot z}\left[\sum_{y} S_{q}(z, y) \gamma_{\sigma} B_{\sigma}^{m_{2}}(y) S_{q}\left(y, x_{\mathrm{op}}\right)\right]\right\} \\
& \cdot \sum_{z^{\prime}}\left\{\left[\sum_{x^{\prime}} S\left(x_{\mathrm{snk}}, x^{\prime}\right) \gamma_{\rho^{\prime}} A_{\rho^{\prime}}^{m_{1}}\left(x^{\prime}\right) S\left(x^{\prime}, z^{\prime}\right)\right] \gamma_{v^{\prime}} e^{-i k \cdot z^{\prime}}\left[\sum_{y^{\prime}} S\left(z^{\prime}, y^{\prime}\right) \gamma_{\sigma^{\prime}} B_{\sigma^{\prime}}^{m_{2}}\left(y^{\prime}\right) S\left(y^{\prime}, x_{\mathrm{src}}\right)\right]\right. \\
& +S\left(x_{\mathrm{snk}}, z^{\prime}\right) \gamma_{v^{\prime}} e^{-i k \cdot z^{\prime}}\left[\sum_{x^{\prime}} S\left(z^{\prime}, x^{\prime}\right) \gamma_{\rho^{\prime}} A_{\rho^{\prime}}^{m_{1}}\left(x^{\prime}\right)\left(\sum_{y^{\prime}} S\left(x^{\prime}, y^{\prime}\right) \gamma_{\sigma^{\prime}} B_{\sigma^{\prime}}^{m_{2}}\left(y^{\prime}\right) S\left(y^{\prime}, x_{\mathrm{src}}\right)\right)\right] \\
& + \text { other } 4 \text { permutations }\}
\end{aligned}
$$

${ }^{1}$ We ignored the multiple photon single link interactions in the lattice simulations presented here so the current is not completely conserved, and the answer not correct. However, these contributions turned out to be very small when we added them. 
Note that the sum over $x, y$ can be independent. This means we can do the sum independently with sequential source method. Also, because of this independence, we can store the results in memory and reuse them for different combination of $m_{1}, m_{2}$. Thus, the number of inversion associated with the fermion loop is only proportion to $M$, but the number of samples grows as $M^{2}$. We call this the $A-B$ method because seperate $A$ and $B$ stochastic variables are being used for the stochastic left and right of the exact photon.

In above formula, we use a point source at all external positions $x_{\mathrm{src}}, x_{\mathrm{snk}}, x_{\mathrm{op}}$ to make the formula more clear. In our simulation code, we use wall sources with half lattice momentum at $x_{\text {src }}$ and $x_{\text {snk }}$. We use random wall source at $x_{\mathrm{op}}$ to implement the fermion loop. We use $S$ to denote the number of random wall sources.

\section{QED Light by Light Simulations}

As a test, we replace the quark loop by a muon loop. The QED LbL results are in Figure 5.
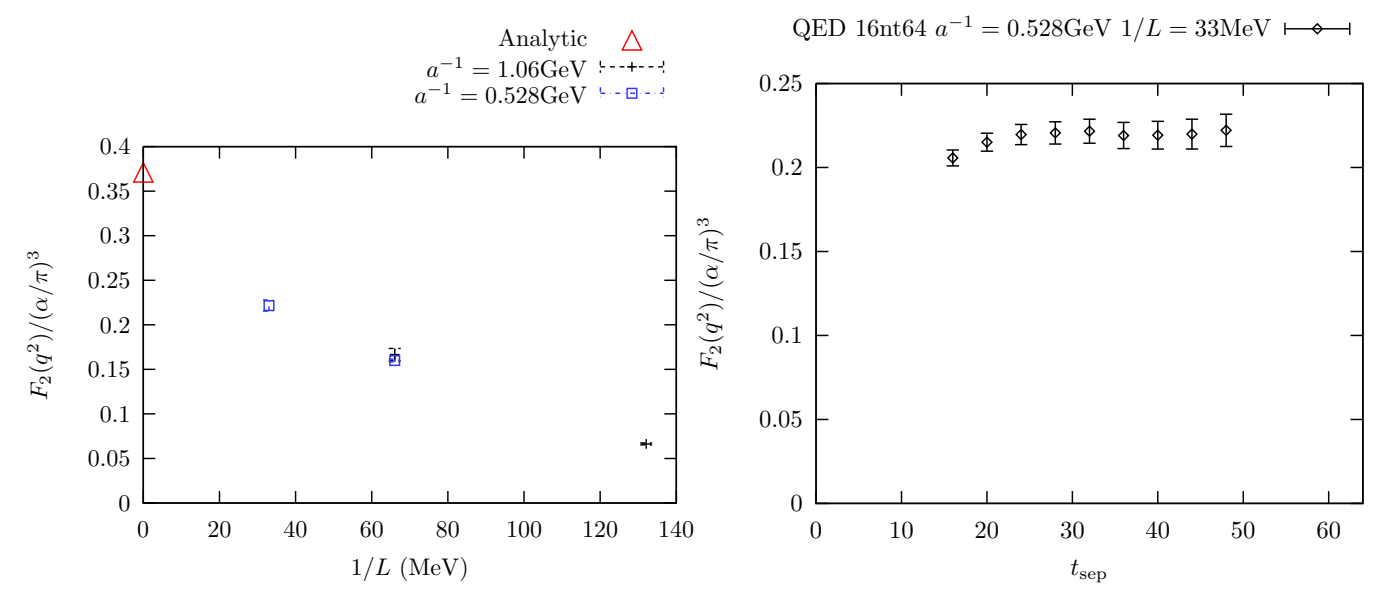

Figure 5: The simulations were done in finite volume with momentum transfer $q=2 \pi / L$. The muon mass $m_{\mu}=105 \mathrm{MeV}$ is used to set the lattice spacing $a$. (L) Finite volume effect on $F_{2}$ : Lattice sizes are $16^{3} \times 64$, $8^{3} \times 32$ with $L_{s}=8$ and $t_{\mathrm{sep}} / 2=t_{\mathrm{snk}}-t_{\mathrm{op}}=t_{\mathrm{op}}-t_{\mathrm{src}}=T / 4$. (R) Excited states effect on $F_{2}$ : Lattice size is $16^{3} \times 64$ with $L_{s}=8$.

\section{Conclusions}

We have investigated some different choices allowed in this computational approach. The results are summarized in the Table 1 . We find that there are three major factors that affect the results.

Averaging over different combinations of the $A, B$ stochastic electromagnetic field reduces the statistical errors, limited only by the memory of the machine. The amount of work is proportion to $M$ but the amount of statistics is roughly proportion to $M^{2}$ as is indicated by the equal variance in the first and second rows in Table 1.

The random wall source at the location of the external current works very well for large lattices. 
We use anti-periodic boundary condition in $z$ direction for the muon propagator and set the momenta of initial and final muon to be $\pm \pi / L$. Using these symmetric kinematics significantly reduces the statistical error as both the initial and the final state are the lowest energy state possible, so the noise to signal ratio does not increase exponentially as thetime separation between muon source and sink grows. Note that this is different from a pion initial/final state, in which case anti-periodic boundary will not help us much since the noise will always decay with the rate of a stationary pion.

$\begin{array}{cccccc}\text { Lattice Size } & t_{\text {sep }} & m_{\mu} a & \frac{\text { Result } \pm \text { Err }}{(\alpha / \pi)^{3}} & N \times S \times M^{2} \text { confs } & \frac{\sqrt{\operatorname{Var}}}{(\alpha / \pi)^{3}} \\ 16^{3} \times 64 & 32 & 0.2 & 0.2228 \pm 0.0046 & 548 \times 18 \times 12^{2} & 5.5 \\ 16^{3} \times 64 & 32 & 0.2 & 0.1962 \pm 0.0368 & 1024 \times 18 \times 1^{2} & 5.0 \\ 16^{3} \times 64 \text { (point src) } & 32 & 0.2 & 0.232 \pm 0.033 & 1508 \times 12 \times 6^{2} & 28.4 \\ 16^{3} \times 64 & 32 & 0.1 & 0.1666 \pm 0.0069 & 88 \times 18 \times 12^{2} & 3.3 \\ 16^{3} \times 64\left(\mathbf{p}_{1}=0\right) & 32 & 0.1 & 0.2278 \pm 0.0265 & 285 \times 36 \times 24^{2} & 64.4\end{array}$

Table 1: $M$ stands for the number of stochastic $A$ or $B$ fields, $S$ stands for the number of random wall sources that we use to calculate the external current. The calculation is repeated $N$ times. $\sqrt{\operatorname{Var}}=\operatorname{Err} \times \sqrt{N \times S \times M^{2}}$ stands for the projected variance deduced from to the statistical uncertainty of the averaged result and the total number of samples.

\section{Acknowledgement}

I would like to thank my advisor Prof. Norman Christ and my other collaborators Thomas Blum, Masashi Hayakawa, Tomomi Ishikawa, Taku Izubuchi, Christoph Lehner, Norikazu YAMADA. I would also like to thank RBRC for BG/Q computer. This work was support in part by US DOE grant DE-SC0011941.

\section{References}

[1] G.W. Bennett et al. Final Report of the Muon E821 Anomalous Magnetic Moment Measurement at BNL. Phys.Rev., D73:072003, 2006, hep-ex/0602035.

[2] Thomas Blum, Saumitra Chowdhury, Masashi Hayakawa, and Taku Izubuchi. Hadronic light-by-light scattering contribution to the muon anomalous magnetic moment from lattice QCD. 2014, 1407.2923.

[3] Thomas Blum, Achim Denig, Ivan Logashenko, Eduardo de Rafael, B. Lee Roberts, et al. The Muon (g-2) Theory Value: Present and Future. 2013, 1311.2198.

[4] Zohreh Davoudi and Martin J. Savage. Finite-Volume Electromagnetic Corrections to the Masses of Mesons, Baryons and Nuclei. Phys.Rev., D90:054503, 2014, 1402.6741.

[5] Masashi Hayakawa, Thomas Blum, Taku Izubuchi, and Norikazu Yamada. Hadronic light-by-light scattering contribution to the muon g-2 from lattice QCD: Methodology. PoS, LAT2005:353, 2006, hep-lat/0509016.

[6] Joaquim Prades, Eduardo de Rafael, and Arkady Vainshtein. Hadronic Light-by-Light Scattering Contribution to the Muon Anomalous Magnetic Moment. 2009, 0901.0306. 